PROCEEDINGS OF THE AMERICAN MATHEMATICAL SOCIETY

Volume 127, Number 3, March 1999, Pages 875-880

S 0002-9939(99)04051-4

\title{
ON THE NON-PRODUCTIVITY OF NORMALITY IN MOORE SPACES
}

\author{
H. COOK AND G. M. REED
}

(Communicated by Franklin D. Tall)

\begin{abstract}
Under Martin's Axiom and the denial of the Continuum Hypothesis, the authors give examples of normal Moore spaces whose squares are not normal.
\end{abstract}

\section{INTRODUCTION}

The question of whether the square of a normal Moore space must be normal was probably first raised by F. B. Jones soon after the publication of the normal Moore space conjecture in [Jo1937]. In any event it was a question often presented to his students in the 1950's and 1960's. Cook and Fitzpatrick showed in [CF1968] that if normality is preserved under finite products in Moore spaces, then it is preserved under countable products (a result which also follows from [K1948], since normal Moore spaces are perfectly normal). In [C1968], Cook established that Moore spaces whose squares are normal must be continuously semi-metrizable. Alster and Przymusiński in [AP1976] showed that under MA, if $X$ is a co-metrizable $T_{2}$-space (i.e., there exists a weaker separable metric topology $\delta$ on $X$ such that if $x \in U$ where $U$ is open in $X$, then there exists open $V$ in $X$ such that $x \in V$ and the closure of $V$ with respect to $\delta$ is contained in $U$ ) and the cardinality of $X<\mathfrak{c}$, then $X^{\omega}$ is normal. Using this result and the result from [RZ1976] that each normal Moore space of cardinality $X \leq \mathfrak{c}$ is submetrizable (i.e., admits a one-to-one continuous map onto a metric space), Reed observed in [R1975] that under MA, the product question has an affirmative answer for locally compact Moore spaces of cardinality $<\mathfrak{c}$. This result was in turn extended by Alster and Przymusiński (see [P1977]) to show that under MA, the product question has an affirmative answer for locally compact separable Moore spaces. Recall that from [FT1966] and [F11974], under $\mathbf{V}=\mathbf{L}$, all normal locally compact Moore spaces are metrizable.

The above results led Reed to conjecture in [R1975] that (1) normality is productive for locally compact Moore spaces in ZFC, and (2) normality is productive for all Moore spaces of cardinality $<\mathfrak{c}$ under MA. The first conjecture remains open, but the authors of this paper have independently now shown that the second conjecture is false. The first examples were due to Cook, who in 1976 established

Received by the editors March 6, 1991.

1991 Mathematics Subject Classification. Primary 54E30, 54D15, 54A35; Secondary 54B10, $54 \mathrm{~A} 10$.

Key words and phrases. Moore spaces, normality, products, Martin's Axiom, intersection topology.

(C)1999 American Mathematical Society 
a complex theory linking the various concepts of continuously semi-metrizability, co-metrizability, and special developments for Moore spaces. He used this theory to provide a necessary condition for the product of a normal Moore space to be normal, and then under $\mathbf{M A}+\neg \mathbf{C H}$ demonstrated the existence of normal Moore spaces without this condition. Although Cook's work was available in preprint [C1976], his examples were never published. In [R1986], Reed studied the class of spaces formed by the intersection topology with respect to subsets of the real line and the order topology on $\omega_{1}$, and noted that under $\mathbf{M A}+\neg \mathbf{C H}$, Moore spaces over members of this class (in the sense of [R1974a]) are normal but not co-metrizable. Hence, Reed raised the question as to whether such normal Moore spaces would fail to have normal squares. Indeed, prior to publication of [R1986], Reed showed this was the case and presented the new class of normal Moore spaces whose squares are not normal at the 1986 Prague Topology Symposium. The proof that the squares of these spaces are not normal is by direct demonstration of two disjoint closed sets which cannot be separated; furthermore, Reed observed that the same simple technique can be used to show the squares of Cook's original spaces are not normal. Thus, the two authors would like to take this opportunity to unify their examples and jointly to place them in the literature.

\section{A Moore SPACE Machine}

In [R1974a] and [R1974b], Reed developed construction techniques which associate Moore spaces to first countable spaces. These techniques and their variations have since been frequently used to provide counterexamples in the theory of Moore spaces. The following construction is the simplest of these techniques; it is merely the first stage in an infinitely iterated process for the other constructions. The description given here is adapted for the applications at hand.

Let $\mathbf{R}$ denote the real numbers, $\mathbf{I}$ the set of integers, $\mathbf{N}$ the non-negative integers, and $\mathbf{N}^{+}$the set of positive integers. Finally, let $\mathbf{F}$ denote $\left\{1 / n: n \in \mathbf{N}^{+}\right\}$.

The construction $\mathcal{M}$. Let $X$ denote a first countable $T_{2}$-space. For each $x \in X$, denote by $\left\{U_{n}(x)\right\}$ a non-increasing sequence of open sets in $X$ which forms a local base at $x$ in $X$.

Let $\mathcal{M}(X)=(X \times\{0\}) \cup(X \times \mathbf{F})$. Denote by $x_{0}$ each element $(x, 0)$ of $X \times\{0\}$ and by $x_{n}$ each element $(x, 1 / n)$ of $X \times \mathbf{F}$.

Now, for each $x_{m}$ in $\mathcal{M}(X)$ and $n \in \mathbf{N}^{+}$, define the basic open set $G_{n}\left(x_{m}\right)$ as follows:

$$
\begin{aligned}
& \text { if } m>0, \quad G_{n}\left(x_{m}\right)=\left\{x_{m}\right\}, \\
& \text { if } m=0, \quad G_{n}\left(x_{m}\right)=\left\{x_{m}\right\} \cup \bigcup\left\{U_{j}(x) \times\{1 / j\}: j \geq n\right\} .
\end{aligned}
$$

It follows that $\left\{\mathcal{G}_{n}\right\}$, where for each $n, \mathcal{G}_{n}=\left\{G_{j}(p): p \in \mathcal{M}(X)\right.$ and $\left.j \geq n\right\}$, is a development for the Moore space $\mathcal{M}(X)$.

\section{A normal Moore space whose square is not normal}

For each $n \in \mathbf{N}^{+}$, let $\mathcal{B}_{n}=\left\{\left(i / 2^{n},(i+1) / 2^{n}\right) \subseteq \mathbf{R}: i \in \mathbf{I}\right\}$. Under $\mathbf{M A}+\neg \mathbf{C H}$, let $X$ denote a $Q$-set on the real line with the inherited topology such that for each $n, \mathcal{B}_{n}$ covers $X$. For each $x \in X$ and $n \in \mathbf{N}^{+}$, let $U_{n}(x)$ denote $B_{x} \cap X$, where $B_{x}$ is the element of $\mathcal{B}_{n}$ containing $x$. 
Consider the space $Z=\mathcal{M}(X)$. By standard arguments (see [T1969]), $Z$ is a normal Moore space.

Let $Z^{\prime}$ denote a new topology on the points of $Z$, where for each $x_{m}$ in $Z^{\prime}$ and $n \in \mathbf{N}^{+}$, basic open sets $G_{n}^{\prime}\left(x_{m}\right)$ are defined as follows:

$$
\begin{aligned}
& \text { if } m>0, \quad G_{n}^{\prime}\left(x_{m}\right)=\left\{x_{m}\right\}, \\
& \text { if } m=0, \quad G_{n}^{\prime}\left(x_{m}\right)=\left\{x_{m}\right\} \cup \bigcup\left\{\left(U_{j}(x) \backslash\{x\}\right) \times\{1 / j\}: j \geq n\right\} .
\end{aligned}
$$

Clearly, $Z^{\prime}$ is also a normal Moore space, since any two subsets of $X \times\{0\}$ which could be separated in $Z$ can also be separated in $Z^{\prime}$. However, the square of $Z^{\prime}$ is trivially not normal. Consider $H=\left\{\left(x_{0}, x_{0}\right): x \in X\right\}$ and $K=\left\{\left(x_{0}, x_{n}\right): x \in X\right.$ and $\left.n \in \mathbf{N}^{+}\right\}$. Clearly $H$ and $K$ are mutually separated in $Z^{\prime} \times Z^{\prime}$, i.e., they are disjoint, no point of $H$ is a limit point of $K$ and no point of $K$ is a limit point of $H$. From [M1962] (Theorem 153), each two mutually separated sets in a normal Moore space can be separated by open sets. Hence suppose $Z^{\prime} \times Z^{\prime}$ is normal and let $V_{H}$ and $V_{K}$ be disjoint open sets in $Z^{\prime} \times Z^{\prime}$ such that $H \subseteq V_{H}$ and $K \subseteq V_{K}$. Since $X$ is uncountable, there exists an uncountable subset $Y$ of $X$ and $n \leq m \in \mathbf{N}^{+}$ such that for all $x \in Y, G_{n}^{\prime}\left(x_{0}\right) \times G_{n}^{\prime}\left(x_{0}\right) \subseteq V_{H}$ and $G_{m}^{\prime}\left(x_{0}\right) \times\left\{\left(x_{n}\right)\right\} \subseteq V_{K}$. Now, there exists $z \in Y$ such that $z$ is a limit point of $Y$ in $X$. It follows that there exists $y \in Y$ with $y \neq z$ such that $z \in U_{m}(y)$ and thus $z \in U_{n}(y)$. Let $z^{\prime} \in U_{m}(y) \cap U_{m}(z)$ such that $z^{\prime} \neq z$. Then, $\left(z_{m}^{\prime}, z_{n}\right) \in G_{n}^{\prime}\left(y_{0}\right) \times G_{n}^{\prime}\left(y_{0}\right) \subseteq V_{H}$ and $\left(z_{m}^{\prime}, z_{n}\right) \in G_{m}^{\prime}\left(z_{0}\right) \times\left\{\left(z_{n}\right)\right\} \subseteq V_{K}$.

Note. Of course, a tangent disc space over $X$ with points in the upper plane isolated, and vertical lines removed from tangent disc neighborhoods for points on the $x$-axis would work just as well. We complicate the space here for simplification of future results.

\section{A normal SeParable Moore space whose Square is not normal}

To achieve the desired result, let us now embed the space $Z^{\prime}$ above in a normal separable Moore space $Z$ ". We will do so by simply "sewing" disjoint spaces $T_{n}$ onto each "level" of $X \times \mathbf{F}$ in $Z^{\prime}$. Each space $T_{n}$ is a copy of a separable subspace $T$ of $Z^{\prime}$.

Consider the construction of $Z^{\prime}$. Let $L$ denote a countable dense subset of the $Q$-set $X$, and consider $T=(X \times\{0\}) \cup(L \times \mathbf{F})$ as a subspace of $Z^{\prime}$. Clearly, $T$ is a normal separable Moore space.

For each $x_{0} \in X \times\{0\}$ and $n \in \mathbf{N}^{+}$, let $G_{n}^{T}\left(x_{0}\right)=G_{n}^{\prime}\left(x_{0}\right) \cap T$, and let $D_{n}\left(x_{0}\right)$ denote an open set in $T$ such that $\left(U_{n}(x) \backslash\{x\}\right) \times\{0\} \subseteq D_{n}\left(x_{0}\right), D_{n}\left(x_{0}\right) \subseteq U_{n}(x) \times$ $(\mathbf{F} \cup\{0\})$, and $x_{0} \notin \overline{D_{n}\left(x_{0}\right)}$.

(*) Note that if $Y \subseteq X \times\{0\}$ and $D=\bigcup\left\{D_{n}(x): x \in Y\right\}$, then $\bar{D}=D$ in $T$.

For each $n \in \mathbf{N}^{+}$, let $T_{n}$ denote a copy of $T$ disjoint from $Z^{\prime}$ such that if $n_{1} \neq n_{2}$, then $T_{n_{1}} \cap T_{n_{2}}=\varnothing$. Also for each $n$, let $\pi_{n}$ denote the identification mapping from $T$ to $T_{n}$, and for each $Y \subseteq T$, let $\pi(Y)$ denote $\bigcup\left\{\pi_{n}(Y): n \in \mathbf{N}^{+}\right\}$. Now, let $Z^{\prime \prime}=Z^{\prime} \cup \pi(L \times \mathbf{F})$. 
For each $z \in Z^{\prime \prime}$ and $n \in \mathbf{N}^{+}$, define the basic open set $G_{n}^{\prime \prime}(z)$ for $z$ in $Z^{\prime \prime}$ as follows:

$$
\begin{array}{rlrl}
\text { if } z \in \pi(L \times \mathbf{F}), & & G_{n}^{\prime \prime}(z) & =\{z\}, \\
\text { if } z=x_{m} \in Z^{\prime} \text { and } m>0, & G_{n}^{\prime \prime}(z) & =\left\{x_{m}\right\} \cup\left(\pi_{m}\left(G_{n}^{T}\left(x_{0}\right)\right) \backslash\left\{\pi_{m}\left(x_{0}\right)\right\}\right), \\
\text { if } z=x_{m} \in Z^{\prime} \text { and } m=0, & G_{n}^{\prime \prime}(z) & =G_{n}^{\prime}\left(x_{m}\right) \cup \\
& & \bigcup\left\{\pi_{j}\left(D_{j}\left(x_{0}\right)\right) \backslash \pi_{j}\left(U_{j}(x) \times\{0\}\right): j \geq n\right\} .
\end{array}
$$

It follows immediately that $Z^{\prime \prime}$ is a separable Moore space and that $Z^{\prime}$ is a closed subspace of $Z^{\prime \prime}$. To see that $Z^{\prime \prime}$ is normal, let $H$ and $K$ denote disjoint closed subsets of $Z^{\prime \prime}$. Without loss of generality let $H=H_{0} \cup H_{F}$ and $K=H_{0} \cup K_{F}$, where $H_{0}, K_{0} \subseteq X \times\{0\}$ and $H_{F}, K_{F} \subseteq X \times \mathbf{F}$. Clearly, since $Z^{\prime}$ is normal, there exists an open set $V_{0}$ in $Z^{\prime \prime}$ containing $H_{0}$ such that $V_{0}$ is the union of basic open sets for elements of $H_{0},\left((X \times\{0\}) \backslash H_{0}\right) \cap V_{0}=\varnothing$, and $\left((X \times\{0\}) \backslash H_{0}\right) \cap \bar{V}_{0}=\varnothing$. Hence by application of the property $(*)$ above, it follows that $\bar{V}_{0}=V_{0}$ in $Z^{\prime \prime}$ and $\bar{V}_{0} \cap K=\varnothing$. Now, by construction and the normality of $T$, for all $n \in \mathbf{N}^{+}$, there exists an open set $V_{n}$ in $Z^{\prime \prime}$ such that $H_{n}=H_{F} \cap X \times\{1 / n\} \subseteq V_{n}$ and $\bar{V}_{n} \cap K=\varnothing$. By symmetry, it follows that $K$ is also the union of countably many sets, each of which can be separated by open sets from $H$. Thus, $H$ and $K$ can be separated by open sets in $Z^{\prime \prime}$.

\section{A Class of normal Moore Spaces WHOSE SQUARES ARE NOT NORMAL}

If $\mathcal{T}_{1}$ and $\mathcal{T}_{2}$ are topologies defined on the set $X$, then $\mathcal{T}$ is the intersection topology w.r.t. $\mathcal{T}_{1}$ and $\mathcal{T}_{2}$ defined on $X$, where $\left\{U_{1} \cap U_{2}: U_{1} \in \mathcal{T}_{1}\right.$ and $\left.U_{2} \in \mathcal{T}_{2}\right\}$ is a basis for $\mathcal{T}$. In [R1986], Reed studied the class $\mathcal{C}$ of spaces $(X, \mathcal{T})$ where $X=\left\{x_{\alpha}: \alpha<\omega_{1}\right\} \subseteq \mathbf{R}$ and $\mathcal{T}$ is the intersection topology w.r.t. the inherited real line topology $\mathcal{T}_{R}$ on $X$ and an order topology $\mathcal{T}_{\omega_{1}}$ on $X$ of type $\omega_{1}$. He showed that each member of $\mathcal{C}$ is a completely regular, first countable, submetrizable, collectionwise Hausdorff, pseudo-normal, countably metacompact space with a base of countable order and a $G_{\delta}$-diagonal, but which is neither $\theta$-refinable, subparacompact, metaLindelöf, nor locally compact. Furthermore, he showed that there exists a non-normal member of $\mathcal{C}$ in $\mathbf{Z F C}$, and a collectionwise normal, $\omega_{1}$-compact, nonperfect member of $\mathcal{C}$ under $\mathbf{C H}$. Finally, Reed noted that under $\mathbf{M A}+\neg \mathbf{C H}$, each member of $\mathcal{C}$ is perfect, whereas Kunen in [K1986] later showed that no member of $\mathcal{C}$ is both perfect and normal, yet there exist models of set theory in which some members of $\mathcal{C}$ are perfect and others are normal.

Notation. If $(X, \mathcal{T}) \in \mathcal{C}$, let $\mathcal{B}_{R}$ denote a countable basis for $\mathcal{T}_{R}$, and for each $x_{\alpha} \in X$, let $\left\{B_{i}\left(x_{\alpha}\right): i \in \mathbf{N}^{+}\right\}$denote a fixed decreasing local base for $x_{\alpha}$ in $\left(X, \mathcal{T}_{R}\right)$ where for each $i, B_{i}\left(x_{\alpha}\right) \in \mathcal{B}_{R}$. For each $x_{\alpha} \in X$ such that $\alpha$ is a limit ordinal in $\omega_{1}$, let $\left\{\alpha_{i}: i \in \mathbf{N}^{+}\right\}$denote a fixed increasing sequence converging to $\alpha$ in $\omega_{1}$. If $x_{\alpha} \in X$ and $\alpha$ is a limit ordinal, $\left\{U_{i}\left(x_{\alpha}\right)=B_{i}\left(x_{\alpha}\right) \cap\left\{x_{\beta}: \alpha_{i}<\beta \leq \alpha\right\}: i \in \mathbf{N}^{+}\right\}$ denotes a fixed local base for $x_{\alpha}$ in $(X, \mathcal{T})$. If $x_{\alpha} \in X$ and $\alpha$ is a non-limit ordinal, then $\left\{U_{i}\left(x_{\alpha}\right)=\left\{\left\{x_{\alpha}\right\}\right\}: i \in \mathbf{N}^{+}\right\}$denotes a fixed local base for $x_{\alpha}$ in $(X, \mathcal{T})$.

Now, let $X=(X, \mathcal{T}) \in \mathcal{C}$, and consider the Moore space $\mathcal{M}(X)$. As above, under MA $+\neg \mathbf{C H}, \mathcal{M}(X)$ is normal. However, we will show that the square of $\mathcal{M}(X)$ is never normal. 
Consider $H=\left\{\left(\left(x_{\alpha}\right)_{0},\left(x_{\alpha}\right)_{0}\right): x_{\alpha} \in X\right\}$ and $K=\left\{\left(\left(x_{\alpha}\right)_{0},\left(x_{\beta}\right)_{n}\right): n \in \mathbf{N}^{+}\right.$, $x_{\alpha} \in X$ and $\left.x_{\beta} \in B_{n}\left(x_{\alpha}\right) \backslash U_{n}\left(x_{\alpha}\right)\right\}$. As before, $H$ and $K$ are mutually separated in $\mathcal{M}(X) \times \mathcal{M}(X)$, hence if we suppose that $\mathcal{M}(X) \times \mathcal{M}(X)$ is normal there exist disjoint open sets $V_{H}$ and $V_{K}$ in $\mathcal{M}(X) \times \mathcal{M}(X)$ such that $H \subseteq V_{H}$ and $K \subseteq V_{K}$. By repeated uses of the Pressing Down Lemma, it follows that there exists a stationary subset $S$ of $\omega_{1}, B \in \mathcal{B}$, and $n<m \in \mathbf{N}^{+}$such that for all $x_{\alpha}$ where $\alpha \in S$,

$$
G_{n}\left(\left(x_{\alpha}\right)_{0}\right) \times G_{n}\left(\left(x_{\alpha}\right)_{0}\right) \subseteq V_{H},
$$

there exists $x_{\alpha^{\prime}}$ such that $\alpha<\alpha^{\prime}$ and $x_{\alpha^{\prime}} \in B_{n}\left(x_{\alpha}\right) \backslash U_{n}\left(x_{\alpha}\right)$,

$$
\begin{gathered}
G_{m}\left(\left(x_{\alpha}\right)_{0}\right) \times\left\{\left\{\left(x_{\alpha^{\prime}}\right)_{n}\right\}\right\} \subseteq V_{K}, \text { and } \\
B_{m}\left(x_{\alpha}\right)=B .
\end{gathered}
$$

Now, since $S$ is stationary, it follows by construction that there exist $\alpha, \gamma \in S$ such that $\left(\left(x_{\alpha}\right)_{m},\left(x_{\alpha^{\prime}}\right)_{n}\right) \in G_{n}\left(\left(x_{\gamma}\right)_{0}\right) \times G_{n}\left(\left(x_{\gamma}\right)_{0}\right) \subseteq V_{H}$ and $\left(\left(x_{\alpha}\right)_{m},\left(x_{\alpha^{\prime}}\right)_{n}\right) \in$ $G_{m}\left(\left(x_{\alpha}\right)_{0}\right) \times\left\{\left\{\left(x_{\alpha^{\prime}}\right)_{n}\right\}\right\} \subseteq V_{K}$. Thus $\mathcal{M}(X) \times \mathcal{M}(X)$ is not normal.

\section{BEYOND MA $+\neg \mathbf{C H}$}

Define a Moore space $S$ to be a sequence space provided $S=H \cup K$, where $H$ is discrete, each point of $K$ is isolated, and basic open neighborhoods for points $x$ in $H$ consist of $x$ together with the tail of a sequence chosen from $K$. Normal Moore sequence spaces which are not collectionwise Hausdorff exist under $\mathbf{M A}+\neg \mathbf{C H}$ [T1969], but the existence of such spaces is also consistent with GCH [DS1979] (see [T1984]). Using the techniques above, Reed has recently shown that the existence of a normal Moore sequence space that is not collectionwise Hausdorff implies the existence of a normal Moore space whose square is not normal. This result will appear as part of a study of Moore sequence spaces in $[R \infty]$.

\section{Questions}

Question 1. Must the square of a normal, locally compact Moore space be normal?

Question 2. Is it consistent with $\mathbf{Z F C}$ that a normal Moore of cardinality $<\mathfrak{c}$ is co-metrizable if and only if its square is normal?

Question 3. Can the normal Moore spaces in section 5 be embedded in normal, separable Moore spaces?

The normal Moore spaces in section 5 can be embedded in separable Moore spaces by methods similar to those in section 4 . Of course, the normal Moore space from [Fl1982] under $\mathbf{C H}$ cannot be embedded in a normal, separable Moore space, since all such spaces are metrizable under CH. See [P1977] and [R1990] for further details related to the above questions.

Question 4. Does the existence of a normal Moore space (not necessarily a sequence space) which is not collectionwise Hausdorff imply the existence of a normal Moore space whose square is not normal?

Question 5. Under $\mathbf{V}=\mathbf{L}$, is the square of each normal Moore space normal? For starters, is the square of Fleissner's normal non-metrizable Moore space from CH in [Fl1982] normal? 


\section{ADDED IN PROOF}

The second author has now answered Question 1 in the negative.

\section{REFERENCES}

[AP1976] K. Alster and T. C. Przymusiński, Normality and Martin's Axiom, Fund. Math. 91 (1976), 124-130. MR 54:3652

[C1968] H. Cook, Cartesian products and continuous semi-metrics, Proc. of the Arizona State University Top. Conf. (1968), 58-63.

[C1976] Cartesian products and continuous semi-metrics, II, preprint, 1976.

[CF1968] H. Cook and B. Fitzpatrick, Inverse limits of perfectly normal Moore spaces, Proc. Amer. Math. Soc. 19 (1968), 189-192. MR 36:3306

[DS1979] K. J. Devlin and S. Shelah, A note on the normal Moore space conjecture, Canad. J. Math. 31, 241-251. MR 81d:54022

[FT1966] B. Fitzpatrick and D. R. Traylor, Two theorems on metrizability of Moore spaces, Pac. J. Math. 19 (1966), 259-264. MR 34:3535

[Fl1974] W. G. Fleissner, Normal Moore spaces in the constructible universe, Proc. Amer. Math. Soc. 46 (1974), 294-298. MR 50:14682

[Fl1982] _ Normal non-metrizable Moore space from continuum hypothesis or nonexistence of inner models with measurable cardinals, Proc. Nat. Acad. Sci. U.S.A. 79 (1982), 1371-1372. MR 84f: 54040

[Jo1937] F. B. Jones, Concerning normal and completely normal spaces, Bull. Amer. Math. Soc. 43 (1937), 671-677.

[K1948] M. Katetov, Complete normality of Cartesian products, Fund. Math. 35 (1948), 271274. MR 10:315h

[K1986] K. Kunen, On ordinal-metric intersection topologies, Topology Appl. 22 (1986), 315319. MR 87i:54046

[M1962] R. L. Moore, Foundations of point set theory, Amer. Math. Soc. Colloq. Publ. 13, 1932 (revised edition, 1962). MR 27:709

[P1977] T. C. Przymusiński, Normality and separability of Moore spaces, Set-Theoretic Topology, Acad. Press (NY, 1977), 325-337. MR 56:6617

[R1974a] G. M. Reed, On chain conditions in Moore spaces, Gen. Top. and Appl. 4 (1974), 255-267. MR 49:9815

[R1974b] _ On continuous images of Moore spaces, Canad. J. Math. 26 (1974), 1475-1479. MR 53:1530

[R1975] _ On the productivity of normality in Moore spaces, Studies in Topology, Acad. Press (NY, 1975), 479-484. MR 53:1531

[R1986]_ The intersection topology w.r.t. the real line and the countable ordinals, Trans. Amer. Math. Soc. 297 (1986), 509-520. MR 87m:54003

[R1990] _ Set-theoretic problems in Moore spaces, Open Problems in Topology, North Holland (Amsterdam, 1990), 163-181.

$[\mathrm{R} \infty] \quad, \quad Q$-sets, stationary sets in $\omega_{1}$, and normal Moore spaces, to appear.

[RZ1976] G. M. Reed and P. L. Zenor, Metrization of Moore spaces and generalized manifolds, Fund. Math. 91 (1976), 213-220. MR 54:13868

[T1969] F. D. Tall, Set-theoretic consistency results and topological theorems concerning the normal Moore space conjecture and related problems, Ph.D. thesis, University of Wisconsin, 1969; Dissert. Math. 148, 1-53. MR 56:13156

[T1984] _ Normality versus collectionwise normality, Handbook of Set-Theoretic Topology (North Holland, 1984), 685-732. MR 86m:54022

Department of Mathematics, University of Houston, Houston, Texas 77004

St Edmund Hall, Oxford OX1 4AR, England

E-mail address: mike.reed@comlab.ox.ac.uk 